\title{
THE RIEMANN HYPOTHESIS
}

\author{
FRANK VEGA
}

\begin{abstract}
In mathematics, the Riemann Hypothesis is a conjecture that the Riemann zeta function has its zeros only at the negative even integers and complex numbers with real part $\frac{1}{2}$. In 1915, Ramanujan proved that under the assumption of the Riemann Hypothesis, the inequality $\sigma(n)<e^{\gamma} \times n \times \log \log n$ holds for all sufficiently large $n$, where $\sigma(n)$ is the sum-of-divisors function and $\gamma \approx 0.57721$ is the Euler-Mascheroni constant. In 1984, Guy Robin proved that the inequality is true for all $n>5040$ if and only if the Riemann Hypothesis is true. In 2002, Lagarias proved that if the inequality $\sigma(n) \leq H_{n}+\exp \left(H_{n}\right) \times \log H_{n}$ holds for all $n \geq 1$, then the Riemann Hypothesis is true, where $H_{n}$ is the $n^{\text {th }}$ harmonic number. We show certain properties of these both inequalities that leave us to a proof of the Riemann Hypothesis.
\end{abstract}

\section{INTRODUCTION}

As usual $\sigma(n)$ is the sum-of-divisors function of $n$ [2]:

$$
\sum_{d \mid n} d
$$

such that $d \mid n$ means the integer $d$ divides to $n$ while $d \nmid n$ means the integer $d$ does not divide to $n$. Define $f(n)$ to be $\frac{\sigma(n)}{n}$. Say Robins $(n)$ holds provided

$$
f(n)<e^{\gamma} \times \log \log n .
$$

The constant $\gamma \approx 0.57721$ is the Euler-Mascheroni constant, and log is the natural logarithm. Let $H_{n}$ be $\sum_{j=1}^{n} \frac{1}{j}$. Say Lagarias $(n)$ holds provided

$$
\sigma(n) \leq H_{n}+\exp \left(H_{n}\right) \times \log H_{n} .
$$

The importance of these properties is:

Theorem 1.1. If Robins $(n)$ holds for all $n>5040$, then the Riemann Hypothesis is true [6]. If Lagarias $(n)$ holds for all $n \geq 1$, then the Riemann Hypothesis is true [5].

It is known that Robins $(n)$ and Lagarias $(n)$ hold for many classes of numbers $n$. We know this:

Lemma 1.2. If Robins $(n)$ holds for some $n>5040$, then Lagarias $(n)$ holds [5].

Here, they are some other results that we use:

Lemma 1.3. Robins $(n)$ holds for every $n>5040$ that is not divisible by 2 [2]. In general, we know that if a positive integer $n$ satisfies either $\nu_{2}(n) \leq 19, \nu_{3}(n) \leq 12$ or $\nu_{7}(n) \leq 6$, then Robins $(n)$ holds, where $\nu_{p}(n)$ is the $p$-adic order of $n$ : In basic

2010 Mathematics Subject Classification. Primary 11M26; Secondary 11A41.

Key words and phrases. number theory, inequality, sum-of-divisors function, harmonic number, prime. 
number theory, for a given prime number $p$, the p-adic order of a positive integer $n$ is the highest exponent $\nu_{p}$ such that $p^{\nu_{p}}$ divides $n$ [4].

Our goal is to prove our main two theorems:

Theorem 1.4. Robins $(n)$ holds for all $n>5040$ when a prime number $q_{m} \nmid n$ for $q_{m} \leq 47$.

Theorem 1.5. Let $n>5040$ and $n=r \times q_{m}$, where $q_{m} \geq 47$ denotes the largest prime factor of $n$. We prove if Lagarias $(r)$ holds, then Lagarias $(n)$ holds.

Consequently, we finally conclude that

Theorem 1.6. Lagarias $(n)$ holds for all $n \geq 1$ and thus, the Riemann Hypothesis is true.

Proof. Every possible counterexample in Lagarias $(n)$ for $n>5040$ must have that its greatest prime factor $q_{m}$ complies with $q_{m} \geq 47$ because of lemma 1.2 and theorem 1.4. On the one hand, Lagarias $(n)$ has been checked for all $n \leq 5040$ by computer. On the other hand, for all $n>5040$ we have that Lagarias $(n)$ has been recursively verified when its greatest prime factor $q_{m}$ complies with $q_{m} \geq 47$ due to theorems 1.4 and 1.5. Indeed, for every natural number $n>5040$, there is always an integer $s$ such that $n=s \times t, s$ is not divisible by any prime number greater than 47 and $s$ is divisible by all the prime powers of $n$ when the prime factors are lesser than 47 (in some cases, the only chance is that $s$ could be lesser than or equal to 5040). In this way, we have that Lagarias(s) holds using the theorem 1.4 and therefore, with a multiplication of factor by factor we could obtain that Lagarias $(s \times t)$ holds recursively over the theorem 1.5. In addition, we can omit the application of the theorem 1.4 when $s \leq 5040$ and obtain the same result, since we know that Lagarias $(s)$ also holds for every natural number $s \leq 5040$. For example, we can show the number $n=17^{3} \times 19^{3} \times 53 \times 113^{2}>5040$ satisfies Lagarias $(n)$, because of Lagarias $\left(17^{3} \times 19^{3}\right)$ holds by theorem 1.4 and therefore, Lagarias $\left(17^{3} \times 19^{3} \times 53\right)$ holds and next Lagarias $\left(17^{3} \times 19^{3} \times 53 \times 113\right)$ holds and finally Lagarias $\left(17^{3} \times 19^{3} \times 53 \times 113^{2}\right)$ holds using recursively the theorem 1.5 just with a multiplication of factor by factor, where every factor is a prime number $q_{m} \geq 47$ such that $q_{m} \in\{53,113\}$. In conclusion, we show that Lagarias $(n)$ holds for all $n \geq 1$ and therefore, the Riemann Hypothesis is true.

\section{KnOwn Results}

We use the following knowledge:

Lemma 2.1. From the reference [2], we know that:

$$
f(n)<\prod_{q \mid n} \frac{q}{q-1} .
$$

Lemma 2.2. From the reference [3, we know that:

$$
\prod_{k=1}^{\infty} \frac{1}{1-\frac{1}{q_{k}^{2}}}=\zeta(2)=\frac{\pi^{2}}{6} .
$$

Lemma 2.3. From the reference [5], we know that:

$$
\log \left(e^{\gamma} \times(n+1)\right) \geq H_{n} \geq \log \left(e^{\gamma} \times n\right) .
$$




\section{A Central Lemma}

The following is a key lemma. It gives an upper bound on $f(n)$ that holds for all $n$. The bound is too weak to prove $\operatorname{Robins}(n)$ directly, but is critical because it holds for all $n$. Further the bound only uses the primes that divide $n$ and not how many times they divide $n$. This is a key insight.

Lemma 3.1. Let $n>1$ and let all its prime divisors be $q_{1}<\cdots<q_{m}$. Then,

$$
f(n)<\frac{\pi^{2}}{6} \times \prod_{i=1}^{m} \frac{q_{i}+1}{q_{i}} .
$$

Proof. We use that lemma 2.1.

$$
f(n)<\prod_{i=1}^{m} \frac{q_{i}}{q_{i}-1} .
$$

Now for $q>1$,

$$
\frac{1}{1-\frac{1}{q^{2}}}=\frac{q^{2}}{q^{2}-1}
$$

So

$$
\begin{aligned}
\frac{1}{1-\frac{1}{q^{2}}} \times \frac{q+1}{q} & =\frac{q^{2}}{q^{2}-1} \times \frac{q+1}{q} \\
& =\frac{q}{q-1}
\end{aligned}
$$

Then by lemma 2.2

$$
\prod_{i=1}^{m} \frac{1}{1-\frac{1}{q_{i}^{2}}}<\zeta(2)=\frac{\pi^{2}}{6} .
$$

Putting this together yields the proof:

$$
\begin{aligned}
f(n) & <\prod_{i=1}^{m} \frac{q_{i}}{q_{i}-1} \\
& \leq \prod_{i=1}^{m} \frac{1}{1-\frac{1}{q_{i}^{2}}} \times \frac{q_{i}+1}{q_{i}} \\
& <\frac{\pi^{2}}{6} \times \prod_{i=1}^{m} \frac{q_{i}+1}{q_{i}} .
\end{aligned}
$$

\section{A Particular Case}

We prove the Robin's inequality for this specific case:

Lemma 4.1. Given a natural number

$$
n=2^{a_{1}} \times 3^{a_{2}} \times 5^{a_{3}} \times 7^{a_{4}}>5040
$$

such that $a_{1}, a_{2}, a_{3}, a_{4} \geq 0$ are integers, then $\operatorname{Robins}(n)$ holds for $n>5040$. 
Proof. Given a natural number $n=q_{1}^{a_{1}} \times q_{2}^{a_{2}} \times \cdots \times q_{m}^{a_{m}}>5040$ such that $q_{1}, q_{2}, \cdots, q_{m}$ are distinct prime numbers and $a_{1}, a_{2}, \cdots, a_{m}$ are natural numbers, we need to prove

$$
f(n)<e^{\gamma} \times \log \log n
$$

that is true when

$$
\prod_{i=1}^{m} \frac{q_{i}}{q_{i}-1} \leq e^{\gamma} \times \log \log n
$$

according to the lemma 2.1. Given a natural number $n=2^{a_{1}} \times 3^{a_{2}} \times 5^{a_{3}}>5040$ such that $a_{1}, a_{2}, a_{3} \geq 0$ are integers, we have

$$
\prod_{i=1}^{m} \frac{q_{i}}{q_{i}-1} \leq \frac{2 \times 3 \times 5}{1 \times 2 \times 4}=3.75<e^{\gamma} \times \log \log (5040) \approx 3.81 .
$$

However, we know for $n>5040$

$$
e^{\gamma} \times \log \log (5040)<e^{\gamma} \times \log \log n
$$

and therefore, the proof is completed for that case. Hence, we only need to prove the Robin's inequality is true for every natural number $n=2^{a_{1}} \times 3^{a_{2}} \times 5^{a_{3}} \times 7^{a_{4}}>5040$ such that $a_{1}, a_{2}, a_{3} \geq 0$ and $a_{4} \geq 1$ are integers. In addition, we know the Robin's inequality is true for every natural number $n>5040$ such that $\nu_{7}(n) \leq 6$, where $\nu_{p}(n)$ is the $p$-adic order of $n$ [4]. Therefore, we need to prove this case for those natural numbers $n>5040$ such that $7^{7} \mid n$. In this way, we have

$$
\prod_{i=1}^{m} \frac{q_{i}}{q_{i}-1} \leq \frac{2 \times 3 \times 5 \times 7}{1 \times 2 \times 4 \times 6}=4.375<e^{\gamma} \times \log \log \left(7^{7}\right) \approx 4.65 .
$$

However, for $n>5040$ and $7^{7} \mid n$, we know that

$$
e^{\gamma} \times \log \log \left(7^{7}\right) \leq e^{\gamma} \times \log \log n
$$

and as a consequence, the proof is completed.

\section{A Better Upper Bound}

Lemma 5.1. For $x \geq 11$, we have

$$
\sum_{q \leq x} \frac{1}{q}<\log \log x+\gamma-0.12
$$

where $q \leq x$ means all the primes lesser than or equal to $x$.

Proof. For $x>1$, we have

$$
\sum_{q \leq x} \frac{1}{q}<\log \log x+B+\frac{1}{\log ^{2} x}
$$

where

$$
B=0.2614972128 \cdots
$$

is the (Meissel-)Mertens constant, since this is a proven result from the article reference [7]. This is the same as

$$
\sum_{q \leq x} \frac{1}{q}<\log \log x+\gamma-\left(C-\frac{1}{\log ^{2} x}\right)
$$


where $\gamma-B=C>0.31$, because of $\gamma>B$. If we analyze $\left(C-\frac{1}{\log ^{2} x}\right)$, then this complies with

$$
\left(C-\frac{1}{\log ^{2} x}\right)>\left(0.31-\frac{1}{\log ^{2} 11}\right)>0.12
$$

for $x \geq 11$ and thus, we finally prove

$$
\sum_{q \leq x} \frac{1}{q}<\log \log x+\gamma-\left(C-\frac{1}{\log ^{2} x}\right)<\log \log x+\gamma-0.12 .
$$

\section{On a Square Free Number}

We recall that an integer $n$ is said to be square free if for every prime divisor $q$ of $n$ we have $q^{2} \nmid n$ [2]. Robins $(n)$ holds for all $n>5040$ that are square free 2]. Let $\operatorname{core}(n)$ denotes the square free kernel of a natural number $n$ [2].

Theorem 6.1. Given a square free number

$$
n=q_{1} \times \cdots \times q_{m}
$$

such that $q_{1}, q_{2}, \cdots, q_{m}$ are odd prime numbers, the greatest prime divisor of $n$ is greater than 7 and $3 \nmid n$, then we obtain the following inequality

$$
\frac{\pi^{2}}{6} \times \frac{3}{2} \times \sigma(n) \leq e^{\gamma} \times n \times \log \log \left(2^{19} \times n\right) .
$$

Proof. This proof is very similar with the demonstration in theorem 1.1 from the article reference [2]. By induction with respect to $\omega(n)$, that is the number of distinct prime factors of $n$ 2. Put $\omega(n)=m$ [2. We need to prove the assertion for those integers with $m=1$. From a square free number $n$, we obtain

$$
\sigma(n)=\left(q_{1}+1\right) \times\left(q_{2}+1\right) \times \cdots \times\left(q_{m}+1\right)
$$

when $n=q_{1} \times q_{2} \times \cdots \times q_{m}[2]$. In this way, for every prime number $q_{i} \geq 11$, then we need to prove

$$
\frac{\pi^{2}}{6} \times \frac{3}{2} \times\left(1+\frac{1}{q_{i}}\right) \leq e^{\gamma} \times \log \log \left(2^{19} \times q_{i}\right) .
$$

For $q_{i}=11$, we have

$$
\frac{\pi^{2}}{6} \times \frac{3}{2} \times\left(1+\frac{1}{11}\right) \leq e^{\gamma} \times \log \log \left(2^{19} \times 11\right)
$$

is actually true. For another prime number $q_{i}>11$, we have

$$
\left(1+\frac{1}{q_{i}}\right)<\left(1+\frac{1}{11}\right)
$$

and

$$
\log \log \left(2^{19} \times 11\right)<\log \log \left(2^{19} \times q_{i}\right)
$$

which clearly implies that the inequality 6.2 is true for every prime number $q_{i} \geq$ 11. Now, suppose it is true for $m-1$, with $m \geq 2$ and let us consider the assertion for those square free $n$ with $\omega(n)=m$ [2]. So let $n=q_{1} \times \cdots \times q_{m}$ be a square free number and assume that $q_{1}<\cdots<q_{m}$ for $q_{m} \geq 11$.

Case 1: $q_{m} \geq \log \left(2^{19} \times q_{1} \times \cdots \times q_{m-1} \times q_{m}\right)=\log \left(2^{19} \times n\right)$. 
By the induction hypothesis we have $\frac{\pi^{2}}{6} \times \frac{3}{2} \times\left(q_{1}+1\right) \times \cdots \times\left(q_{m-1}+1\right) \leq e^{\gamma} \times q_{1} \times \cdots \times q_{m-1} \times \log \log \left(2^{19} \times q_{1} \times \cdots \times q_{m-1}\right)$ and hence

$$
\begin{gathered}
\frac{\pi^{2}}{6} \times \frac{3}{2} \times\left(q_{1}+1\right) \times \cdots \times\left(q_{m-1}+1\right) \times\left(q_{m}+1\right) \leq \\
e^{\gamma} \times q_{1} \times \cdots \times q_{m-1} \times\left(q_{m}+1\right) \times \log \log \left(2^{19} \times q_{1} \times \cdots \times q_{m-1}\right)
\end{gathered}
$$

when we multiply the both sides of the inequality by $\left(q_{m}+1\right)$. We want to show

$$
e^{\gamma} \times q_{1} \times \cdots \times q_{m-1} \times\left(q_{m}+1\right) \times \log \log \left(2^{19} \times q_{1} \times \cdots \times q_{m-1}\right) \leq
$$

$e^{\gamma} \times q_{1} \times \cdots \times q_{m-1} \times q_{m} \times \log \log \left(2^{19} \times q_{1} \times \cdots \times q_{m-1} \times q_{m}\right)=e^{\gamma} \times n \times \log \log \left(2^{19} \times n\right)$. Indeed the previous inequality is equivalent with

$q_{m} \times \log \log \left(2^{19} \times q_{1} \times \cdots \times q_{m-1} \times q_{m}\right) \geq\left(q_{m}+1\right) \times \log \log \left(2^{19} \times q_{1} \times \cdots \times q_{m-1}\right)$ or alternatively

$$
\begin{gathered}
\frac{q_{m} \times\left(\log \log \left(2^{19} \times q_{1} \times \cdots \times q_{m-1} \times q_{m}\right)-\log \log \left(2^{19} \times q_{1} \times \cdots \times q_{m-1}\right)\right)}{\log q_{m}} \geq \\
\frac{\log \log \left(2^{19} \times q_{1} \times \cdots \times q_{m-1}\right)}{\log q_{m}} .
\end{gathered}
$$

From the reference [2], we have if $0<a<b$, then

$$
\frac{\log b-\log a}{b-a}=\frac{1}{(b-a)} \int_{a}^{b} \frac{d t}{t}>\frac{1}{b} .
$$

We can apply the inequality 6.3$)$ to the previous one just using $b=\log \left(2^{19} \times q_{1} \times\right.$ $\left.\cdots \times q_{m-1} \times q_{m}\right)$ and $a=\log \left(2^{19} \times q_{1} \times \cdots \times q_{m-1}\right)$. Certainly, we have

$$
\begin{gathered}
\log \left(2^{19} \times q_{1} \times \cdots \times q_{m-1} \times q_{m}\right)-\log \left(2^{19} \times q_{1} \times \cdots \times q_{m-1}\right)= \\
\log \frac{2^{19} \times q_{1} \times \cdots \times q_{m-1} \times q_{m}}{2^{19} \times q_{1} \times \cdots \times q_{m-1}}=\log q_{m} .
\end{gathered}
$$

In this way, we obtain

$$
\begin{gathered}
\frac{q_{m} \times\left(\log \log \left(2^{19} \times q_{1} \times \cdots \times q_{m-1} \times q_{m}\right)-\log \log \left(2^{19} \times q_{1} \times \cdots \times q_{m-1}\right)\right)}{\log q_{m}}> \\
\frac{q_{m}}{\log \left(2^{19} \times q_{1} \times \cdots \times q_{m}\right)} .
\end{gathered}
$$

Using this result we infer that the original inequality is certainly satisfied if the next inequality is satisfied

$$
\frac{q_{m}}{\log \left(2^{19} \times q_{1} \times \cdots \times q_{m}\right)} \geq \frac{\log \log \left(2^{19} \times q_{1} \times \cdots \times q_{m-1}\right)}{\log q_{m}}
$$

which is trivially true for $q_{m} \geq \log \left(2^{19} \times q_{1} \times \cdots \times q_{m-1} \times q_{m}\right)$ [2].

Case 2: $q_{m}<\log \left(2^{19} \times q_{1} \times \cdots \times q_{m-1} \times q_{m}\right)=\log \left(2^{19} \times n\right)$.

We need to prove

$$
\frac{\pi^{2}}{6} \times \frac{3}{2} \times \frac{\sigma(n)}{n} \leq e^{\gamma} \times \log \log \left(2^{19} \times n\right) .
$$

We know $\frac{3}{2}<1.503<\frac{4}{2.66}$. Nevertheless, we could have

$$
\frac{3}{2} \times \frac{\sigma(n)}{n} \times \frac{\pi^{2}}{6}<\frac{4 \times \sigma(n)}{3 \times n} \times \frac{\pi^{2}}{2 \times 2.66}
$$


and therefore, we only need to prove

$$
\frac{\sigma(3 \times n)}{3 \times n} \times \frac{\pi^{2}}{5.32} \leq e^{\gamma} \times \log \log \left(2^{19} \times n\right)
$$

where this is possible because of $3 \nmid n$. If we apply the logarithm to the both sides of the inequality, then we obtain

$\log \left(\frac{\pi^{2}}{5.32}\right)+(\log (3+1)-\log 3)+\sum_{i=1}^{m}\left(\log \left(q_{i}+1\right)-\log q_{i}\right) \leq \gamma+\log \log \log \left(2^{19} \times n\right)$.

From the reference [2, we note

$$
\log \left(q_{1}+1\right)-\log q_{1}=\int_{q_{1}}^{q_{1}+1} \frac{d t}{t}<\frac{1}{q_{1}} .
$$

In addition, note $\log \left(\frac{\pi^{2}}{5.32}\right)<\frac{1}{2}+0.12$. However, we know

$$
\gamma+\log \log q_{m}<\gamma+\log \log \log \left(2^{19} \times n\right)
$$

since $q_{m}<\log \left(2^{19} \times n\right)$ and therefore, it is enough to prove

$$
0.12+\frac{1}{2}+\frac{1}{3}+\frac{1}{q_{1}}+\cdots+\frac{1}{q_{m}} \leq 0.12+\sum_{q \leq q_{m}} \frac{1}{q} \leq \gamma+\log \log q_{m}
$$

where $q_{m} \geq 11$. In this way, we only need to prove

$$
\sum_{q \leq q_{m}} \frac{1}{q} \leq \gamma+\log \log q_{m}-0.12
$$

which is true according to the lemma 5.1 when $q_{m} \geq 11$. In this way, we finally show the theorem is indeed satisfied.

\section{ROBIN ON DIVISIBILITY}

Theorem 7.1. Robins $(n)$ holds for all $n>5040$ when $3 \nmid n$. More precisely: every possible counterexample $n>5040$ of the Robin's inequality must comply with $\left(2^{20} \times 3^{13}\right) \mid n$.

Proof. We will check the Robin's inequality is true for every natural number $n=$ $q_{1}^{a_{1}} \times q_{2}^{a_{2}} \times \cdots \times q_{m}^{a_{m}}>5040$ such that $q_{1}, q_{2}, \cdots, q_{m}$ are distinct prime numbers, $a_{1}, a_{2}, \cdots, a_{m}$ are natural numbers and $3 \nmid n$. We know this is true when the greatest prime divisor of $n>5040$ is lesser than or equal to 7 according to the lemma 4.1. Therefore, the remaining case is when the greatest prime divisor of $n>5040$ is greater than 7 . We need to prove

$$
f(n)<e^{\gamma} \times \log \log n
$$

that is true when

$$
\frac{\pi^{2}}{6} \times \prod_{i=1}^{m} \frac{q_{i}+1}{q_{i}} \leq e^{\gamma} \times \log \log n
$$

according to the lemma 3.1 . Using the formula 6.1 , we obtain that will be equivalent to

$$
\frac{\pi^{2}}{6} \times \frac{\sigma\left(n^{\prime}\right)}{n^{\prime}} \leq e^{\gamma} \times \log \log n
$$

where $n^{\prime}=q_{1} \times \cdots \times q_{m}$ is the core $(n)$ [2]. However, the Robin's inequality has been proved for all integers $n$ not divisible by 2 (which are bigger than 10) [2]. Hence, 
we only need to prove the Robin's inequality is true when $2 \mid n^{\prime}$. In addition, we know the Robin's inequality is true for every natural number $n>5040$ such that $\nu_{2}(n) \leq 19$, where $\nu_{p}(n)$ is the $p$-adic order of $n$ [4. Consequently, we only need to prove the Robin's inequality is true for all $n>5040$ such that $2^{20} \mid n$ and thus,

$$
e^{\gamma} \times n^{\prime} \times \log \log \left(2^{19} \times \frac{n^{\prime}}{2}\right) \leq e^{\gamma} \times n^{\prime} \times \log \log n
$$

because of $2^{19} \times \frac{n^{\prime}}{2} \leq n$ when $2^{20} \mid n$ and $2 \mid n^{\prime}$. In this way, we only need to prove

$$
\frac{\pi^{2}}{6} \times \sigma\left(n^{\prime}\right) \leq e^{\gamma} \times n^{\prime} \times \log \log \left(2^{19} \times \frac{n^{\prime}}{2}\right) .
$$

According to the formula (6.1) and $2 \mid n^{\prime}$, we have

$$
\frac{\pi^{2}}{6} \times 3 \times \sigma\left(\frac{n^{\prime}}{2}\right) \leq e^{\gamma} \times 2 \times \frac{n^{\prime}}{2} \times \log \log \left(2^{19} \times \frac{n^{\prime}}{2}\right)
$$

which is the same as

$$
\frac{\pi^{2}}{6} \times \frac{3}{2} \times \sigma\left(\frac{n^{\prime}}{2}\right) \leq e^{\gamma} \times \frac{n^{\prime}}{2} \times \log \log \left(2^{19} \times \frac{n^{\prime}}{2}\right)
$$

that is true according to the theorem 6.1 when $3 \nmid \frac{n^{\prime}}{2}$. In addition, we know the Robin's inequality is true for every natural number $n>5040$ such that $\nu_{3}(n) \leq 12$, where $\nu_{p}(n)$ is the $p$-adic order of $n$ [4]. Consequently, we only need to prove the Robin's inequality is true for all $n>5040$ such that $2^{20} \mid n$ and $3^{13} \mid n$. To sum up, the proof is completed.

Theorem 7.2. Robins $(n)$ holds for all $n>5040$ when $5 \nmid n$ or $7 \nmid n$.

Proof. We need to prove

$$
f(n)<e^{\gamma} \times \log \log n
$$

when $\left(2^{20} \times 3^{13}\right) \mid n$. Suppose that $n=2^{a} \times 3^{b} \times m$, where $a \geq 20, b \geq 13,2 \nmid m$, $3 \nmid m$ and $5 \nmid m$ or $7 \nmid m$. Therefore, we need to prove

$$
f\left(2^{a} \times 3^{b} \times m\right)<e^{\gamma} \times \log \log \left(2^{a} \times 3^{b} \times m\right) .
$$

We know

$$
f\left(2^{a} \times 3^{b} \times m\right)=f\left(3^{b}\right) \times f\left(2^{a} \times m\right)
$$

since $f$ is multiplicative [9]. In addition, we know $f\left(3^{b}\right)<\frac{3}{2}$ for every natural number $b$ [9. In this way, we have

$$
f\left(3^{b}\right) \times f\left(2^{a} \times m\right)<\frac{3}{2} \times f\left(2^{a} \times m\right) .
$$

Now, consider

$$
\frac{3}{2} \times f\left(2^{a} \times m\right)=\frac{9}{8} \times f(3) \times f\left(2^{a} \times m\right)=\frac{9}{8} \times f\left(2^{a} \times 3 \times m\right)
$$

where $f(3)=\frac{4}{3}$ since $f$ is multiplicative $[9$. Nevertheless, we have

$$
\frac{9}{8} \times f\left(2^{a} \times 3 \times m\right)<f(5) \times f\left(2^{a} \times 3 \times m\right)=f\left(2^{a} \times 3 \times 5 \times m\right)
$$

and

$$
\frac{9}{8} \times f\left(2^{a} \times 3 \times m\right)<f(7) \times f\left(2^{a} \times 3 \times m\right)=f\left(2^{a} \times 3 \times 7 \times m\right)
$$

where $5 \nmid m$ or $7 \nmid m, f(5)=\frac{6}{5}$ and $f(7)=\frac{8}{7}$. However, we know the Robin's inequality is true for $2^{a} \times 3 \times 5 \times m$ and $2^{a} \times 3 \times 7 \times m$ when $a \geq 20$, since this is 
true for every natural number $n>5040$ such that $\nu_{3}(n) \leq 12$, where $\nu_{p}(n)$ is the $p$-adic order of $n$ [4. Hence, we would have

$$
f\left(2^{a} \times 3 \times 5 \times m\right)<e^{\gamma} \times \log \log \left(2^{a} \times 3 \times 5 \times m\right)<e^{\gamma} \times \log \log \left(2^{a} \times 3^{b} \times m\right)
$$

and

$$
f\left(2^{a} \times 3 \times 7 \times m\right)<e^{\gamma} \times \log \log \left(2^{a} \times 3 \times 7 \times m\right)<e^{\gamma} \times \log \log \left(2^{a} \times 3^{b} \times m\right)
$$

when $b \geq 13$.

Theorem 7.3. Robins $(n)$ holds for all $n>5040$ when a prime number $q_{m} \nmid n$ for $11 \leq q_{m} \leq 47$.

Proof. We know the Robin's inequality is true for every natural number $n>5040$ such that $\nu_{7}(n) \leq 6$, where $\nu_{p}(n)$ is the $p$-adic order of $n$ [4. We need to prove

$$
f(n)<e^{\gamma} \times \log \log n
$$

when $\left(2^{20} \times 3^{13} \times 7^{7}\right) \mid n$. Suppose that $n=2^{a} \times 3^{b} \times 7^{c} \times m$, where $a \geq 20, b \geq 13$, $c \geq 7,2 \nmid m, 3 \nmid m, 7 \nmid m, q_{m} \nmid m$ and $11 \leq q_{m} \leq 47$. Therefore, we need to prove

$$
f\left(2^{a} \times 3^{b} \times 7^{c} \times m\right)<e^{\gamma} \times \log \log \left(2^{a} \times 3^{b} \times 7^{c} \times m\right) .
$$

We know

$$
f\left(2^{a} \times 3^{b} \times 7^{c} \times m\right)=f\left(7^{c}\right) \times f\left(2^{a} \times 3^{b} \times m\right)
$$

since $f$ is multiplicative [9]. In addition, we know $f\left(7^{c}\right)<\frac{7}{6}$ for every natural number $c$ [9. In this way, we have

$$
f\left(7^{c}\right) \times f\left(2^{a} \times 3^{b} \times m\right)<\frac{7}{6} \times f\left(2^{a} \times 3^{b} \times m\right) .
$$

However, that would be equivalent to

$$
\frac{49}{48} \times f(7) \times f\left(2^{a} \times 3^{b} \times m\right)=\frac{49}{48} \times f\left(2^{a} \times 3^{b} \times 7 \times m\right)
$$

where $f(7)=\frac{8}{7}$ since $f$ is multiplicative [9]. In addition, we know

$\frac{49}{48} \times f\left(2^{a} \times 3^{b} \times 7 \times m\right)<f\left(q_{m}\right) \times f\left(2^{a} \times 3^{b} \times 7 \times m\right)=f\left(2^{a} \times 3^{b} \times 7 \times q_{m} \times m\right)$

where $q_{m} \nmid m, f\left(q_{m}\right)=\frac{q_{m}+1}{q_{m}}$ and $11 \leq q_{m} \leq 47$. Nevertheless, we know the Robin's inequality is true for $2^{a} \times 3^{b} \times 7 \times q_{m} \times m$ when $a \geq 20$ and $b \geq 13$, since this is true for every natural number $n>5040$ such that $\nu_{7}(n) \leq 6$, where $\nu_{p}(n)$ is the $p$-adic order of $n$ [4]. Hence, we would have

$$
\begin{aligned}
f\left(2^{a} \times 3^{b} \times 7 \times q_{m} \times m\right) & <e^{\gamma} \times \log \log \left(2^{a} \times 3^{b} \times 7 \times q_{m} \times m\right) \\
& <e^{\gamma} \times \log \log \left(2^{a} \times 3^{b} \times 7^{c} \times m\right)
\end{aligned}
$$

when $c \geq 7$ and $11 \leq q_{m} \leq 47$. 


\section{Proof of Main Theorems}

Theorem 8.1. Robins $(n)$ holds for all $n>5040$ when a prime number $q_{m} \nmid n$ for $q_{m} \leq 47$.

Proof. This is a compendium of the results from the Theorems 7.1, 7.2 and 7.3.

Theorem 8.2. Let $n>5040$ and $n=r \times q_{m}$, where $q_{m} \geq 47$ denotes the largest prime factor of $n$. We prove if Lagarias $(r)$ holds, then Lagarias $(n)$ holds.

Proof. We need to prove

$$
\sigma(n) \leq H_{n}+\exp \left(H_{n}\right) \times \log H_{n} .
$$

We have that

$$
\sigma(r) \leq H_{r}+\exp \left(H_{r}\right) \times \log H_{r}
$$

since Lagarias $(r)$ holds. If we multiply by $\left(q_{m}+1\right)$ the both sides of the previous inequality, then we obtain that

$$
\sigma(r) \times\left(q_{m}+1\right) \leq\left(q_{m}+1\right) \times H_{r}+\left(q_{m}+1\right) \times \exp \left(H_{r}\right) \times \log H_{r} .
$$

We know that $\sigma$ is submultiplicative (that is $\sigma(n)=\sigma\left(q_{m} \times r\right) \leq \sigma\left(q_{m}\right) \times \sigma(r)$ ) [2]. Moreover, we know that $\sigma\left(q_{m}\right)=\left(q_{m}+1\right)$ [2]. In this way, we obtain that

$$
\sigma(n)=\sigma\left(q_{m} \times r\right) \leq\left(q_{m}+1\right) \times H_{r}+\left(q_{m}+1\right) \times \exp \left(H_{r}\right) \times \log H_{r} .
$$

Hence, it is enough to prove that

$$
\begin{aligned}
& \left(q_{m}+1\right) \times H_{r}+\left(q_{m}+1\right) \times \exp \left(H_{r}\right) \times \log H_{r} \\
& \leq H_{n}+\exp \left(H_{n}\right) \times \log H_{n} \\
& =H_{q_{m} \times r}+\exp \left(H_{q_{m} \times r}\right) \times \log H_{q_{m} \times r} .
\end{aligned}
$$

If we apply the lemma 2.3 to the previous inequality, then we could only need to show that

$$
\begin{aligned}
& \left(q_{m}+1\right) \times \log \left(e^{\gamma} \times(r+1)\right)+\left(q_{m}+1\right) \times e^{\gamma} \times(r+1) \times \log \log \left(e^{\gamma} \times(r+1)\right) \\
& \leq \log \left(e^{\gamma} \times q_{m} \times r\right)+e^{\gamma} \times q_{m} \times r \times \log \log \left(e^{\gamma} \times q_{m} \times r\right) .
\end{aligned}
$$

We know this last inequality is true since we can easily check that the subtraction of

with

$$
\log \left(e^{\gamma} \times q_{m} \times r\right)+e^{\gamma} \times q_{m} \times r \times \log \log \left(e^{\gamma} \times q_{m} \times r\right)
$$

$$
\left(q_{m}+1\right) \times \log \left(e^{\gamma} \times(r+1)\right)+\left(q_{m}+1\right) \times e^{\gamma} \times(r+1) \times \log \log \left(e^{\gamma} \times(r+1)\right)
$$

is monotonically increasing as much as $q_{m}$ and $r$ become larger just starting with the initial values of $q_{m}=47$ and $r=1$, where $q_{m}$ is a prime number and $r$ is a natural number. Actually, this evidence seems more obvious when the values of $q_{m}$ and $r$ are incremented much more even for real numbers. Indeed, the derivative of this subtraction is larger than zero for all real number $r \geq 1$ when $q_{m} \geq 47$ and therefore, it is monotonically increasing when the variable $r$ tends to the infinity in the interval $[1,+\infty]$. Since there is nothing that can avoid this increasing behavior since this subtraction is continuous in that interval, then we could state this theorem is always true.

In fact, a function $f(r)$ of a real variable $r$ is monotonically increasing in some interval if the derivative of $f(r)$ is larger than zero and the function $f(r)$ is continuous over that interval [1]. Certainly, the derivative of this subtraction is larger 
than zero over the evaluation of $r$ in $[1,+\infty]$, just because of the impact that has the value of $q_{m} \geq 47$ in the whole differentiation, where we know the derivative of $\log x$ and $\log \log x$ is $\frac{1}{x}$ and $\frac{1}{x \times \log x}$ respectively [8]. Of course, this result is not true for some small values in the range of $1<q_{m}<47$, that's why it's so important this detail. Consequently, if this subtraction is monotonically increasing for the real numbers, then this will be the same when $q_{m} \geq 47$ is a prime number and $r$ is a natural number. In this way, we can claim that Lagarias $(n)$ has been checked for $n=r \times q_{m}$ when Lagarias $(r)$ holds and the largest prime factor $q_{m}$ of $n$ complies with $q_{m} \geq 47$.

\section{ACKNOWLEDGMENTS}

I thank Richard J. Lipton for helpful comments.

\section{REFERENCES}

[1] Glen Anderson, Mavina Vamanamurthy, and Matti Vuorinen. Monotonicity Rules in Calculus. The American Mathematical Monthly, 113(9):805-816, 2006. doi:10.1080/00029890.2006. 11920367

[2] YoungJu Choie, Nicolas Lichiardopol, Pieter Moree, and Patrick Solé. On Robin's criterion for the Riemann hypothesis. Journal de Théorie des Nombres de Bordeaux, 19(2):357-372, 2007. doi: $10.5802 / j \operatorname{tnb} .591$

[3] Harold M. Edwards. Riemann's Zeta Function. Dover Publications, 2001.

[4] Alexander Hertlein. Robin's Inequality for New Families of Integers. Integers, 18, 2018.

[5] Jeffrey C. Lagarias. An Elementary Problem Equivalent to the Riemann Hypothesis. The American Mathematical Monthly, 109(6):534-543, 2002. doi:10.2307/2695443

[6] Guy Robin. Grandes valeurs de la fonction somme des diviseurs et hypothèse de Riemann. $J$. Math. pures appl, 63(2):187-213, 1984.

[7] J. Barkley Rosser and Lowell Schoenfeld. Approximate Formulas for Some Functions of Prime Numbers. Illinois Journal of Mathematics, 6(1):64-94, 1962. doi:10.1215/ijm/1255631807

[8] Murray R. Spiegel, Seymour Lipschutz, and John Liu. Schaum's Outlines: Mathematical Handbook of Formulas and Tables, volume 2. McGraw-Hill New York, 2009.

[9] Robert Vojak. On numbers satisfying Robin's inequality, properties of the next counterexample and improved specific bounds. arXiv preprint arXiv:2005.0930\%, 2020.

CopSonic, 1471 Route de Saint-Nauphary 82000 Montauban, France

E-mail address: vega.frank@gmail.com 